\title{
Dual PD Control Regulation with Nonlinear Compensation for a Ball and Plate System
}

\author{
Sergio Galvan-Colmenares, ${ }^{1}$ Marco A. Moreno-Armendáriz, ${ }^{1}$ José de Jesús Rubio, ${ }^{2}$ \\ Floriberto Ortíz-Rodriguez, ${ }^{3}$ Wen Yu, ${ }^{4}$ and Carlos F. Aguilar-Ibáñez ${ }^{1}$ \\ ${ }^{1}$ Centro de Investigación en Computación, Instituto Politécnico Nacional, Avenida Juan de Dios Bátiz S/N, Colonia Zacatenco, \\ 07738 México, DF, Mexico \\ ${ }^{2}$ Sección de Estudios de Posgrado e Investigación, ESIME Azcapotzalco, Instituto Politécnico Nacional, Avenida de las Granjas No. 682, \\ Colonia Santa Catarina, 02250 México, DF, Mexico \\ ${ }^{3}$ Escuela Superior de Ingenieria Mecánica y Eléctrica, Instituto Politécnico Nacional, Avenida Juan de Dios Bátiz S/N, Zacatenco, \\ 07738 México, DF, Mexico \\ ${ }^{4}$ Departamento de Control Automatico, CINVESTAV, Instituto Politécnico Nacional, 07370 México, DF, Mexico
}

Correspondence should be addressed to José de Jesús Rubio; jrubioa@ipn.mx

Received 11 November 2013; Accepted 4 January 2014; Published 16 April 2014

Academic Editor: Mingcong Deng

Copyright (c) 2014 Sergio Galvan-Colmenares et al. This is an open access article distributed under the Creative Commons Attribution License, which permits unrestricted use, distribution, and reproduction in any medium, provided the original work is properly cited.

\begin{abstract}
The normal proportional derivative (PD) control is modified to a new dual form for the regulation of a ball and plate system. First, to analyze this controller, a novel complete nonlinear model of the ball and plate system is obtained. Second, an asymptotic stable dual PD control with a nonlinear compensation is developed. Finally, the experimental results of ball and plate system are provided to verify the effectiveness of the proposed methodology.
\end{abstract}

\section{Introduction}

The ball and plate system is one of the most popular and important models in control education, and it is a benchmark nonlinear plant [1], because it is more complex than the traditional ball and beam system [2]. The ball and beam system has two degrees of freedom where the ball can roll freely on a rigid plate; see Figure 1. Since the movement of the ball over the plate can reach high speeds, the design of a suitable controller for this system is a major challenge; therefore, these systems are not commonly used in laboratories.

The ball and plate system has low consumer appeal. However, the challenge of balancing is a problem under continuous study for robotics transportation applications, often as extensions of the inverted pendulum project. Therefore, the system can present many challenges and opportunities as an educational tool of the control systems engineering for university students. Thus, this system has enormous potential for advanced control techniques, such as fuzzy control $[3,4]$, classical control [5], robust control [6-10], nonlinear control $[11,12]$, and sliding mode control [13].

There are some reported control algorithms for the ball and plate system in the literature. In [14], a stabilizing switching control scheme is proposed. By the concepts of switchingdriving Lyapunov function and switching-driven stability, a locally asymptotically stabilizing switching controller is obtained. In [15], a linear model and simple PID controller are used for a real-time ball and plate system based on DSpace. On the other hand, intelligent control is suitable when the model of the ball and plate system is not available. In [16], a hierarchical fuzzy control with a genetic algorithm is applied for the adjustment of the membership functions.

From the above controllers, there are two problems for the ball and plate control:

(1) some laboratories use simple controllers such as PD control, and the theory analysis is based on linear models [15]; 
(2) nonlinear controllers for ball and plate system have good theory results; nevertheless, they are complex and rarely used in real applications [15].

In this paper, a novel dual PD control for the ball and plate system is presented. Continuing with the previous results of this research group, on the ball and beam system with two degrees of freedom of [17], a new ball and plate is assembled in [18] to reach the objective of this research. This prototype fulfills the requirements of a real-time control via an embedded system designed by minimal resources. To analyze the closed-loop system (see Figure 2), a new dynamic model of the ball and plate is obtained; therefore, a stability analysis using this novel nonlinear model is developed. To the best of our knowledge, this kind of analysis has not yet been established in the literature. Finally, experimental results are performed to evaluate the theory results.

This paper is organized as follows. In Section 2, the ball and plate dynamic model is introduced. Section 3 introduces dual PD control. In Section 4, the stability analysis of the proposed controller is considered. Ball and beam prototype is shown in Section 5. In Section 6, experimental results of three controllers applied to the ball and beam system are shown. Section 7 presents conclusions and suggests future research directions.

\section{Ball and Plate System}

The new ball and plate system is shown in Figure 1. In this system a ball is placed on a plate where it is allowed to roll with two degrees of freedom. As the two motors turn, they produce a shift in the angles $\theta_{x}$ and $\theta_{y}$, which at the same time cause a variation of position of the ball in $(x, y)$ due to the gravity force. In the absence of friction or other disturbances, the dynamics of the ball and plate system can be obtained by the Lagrangian method $[19,20]$.

The kinetic energy of the system is

$$
\begin{aligned}
E_{c}= & \frac{1}{2} m v_{c d m}^{2}+\frac{1}{2} I_{c d m} w^{2} \\
= & \frac{1}{2} m\left(\dot{x}^{2}+\dot{y}^{2}\right)+\frac{1}{2} m\left(x \dot{\theta}_{x}+y \dot{\theta}_{y}\right)^{2} \\
& +\frac{1}{2} I_{c d m}\left(\frac{\dot{x}^{2}+\dot{y}^{2}}{R}\right)+\frac{1}{2} I_{c d m}\left(\dot{\theta}_{x}^{2}+\dot{\theta}_{y}^{2}\right),
\end{aligned}
$$

where $E_{c}$ is the kinetic energy of the ball; $m$ is the mass of the ball; $I_{c d m}$ is the inertia moment of the ball; $R$ is the radio of the ball; $\sqrt{\dot{x}^{2}+\dot{y}^{2}}$ is the linear velocity of the ball; $x$ and $y$ are the position of the ball on plate; $\theta_{x}$ and $\theta_{y}$ are the angular position of the plate; $w$ is the angular velocity of the ball; and $\sqrt{\dot{\theta}_{x}^{2}+\dot{\theta}_{y}^{2}}$ is the angular velocity of the plate.

On the other hand, potential energy of the ball due to the gravity is given by

$$
E_{p}=m G\left(x \sin \left(\theta_{x}\right)+y \sin \left(\theta_{y}\right)\right),
$$

where $E_{p}$ is the potential energy of the ball and $G$ is the acceleration due to gravity.

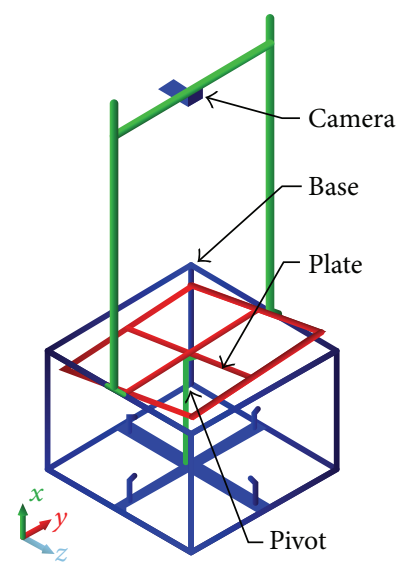

(a)

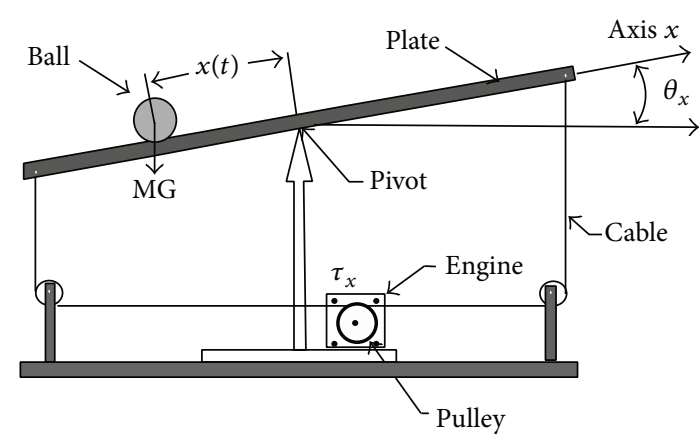

(b)

FIgURE 1: Ball and plate system.

From (1) and (2), the Lagrange equation is

$$
\begin{aligned}
L= & \frac{1}{2} m\left(\dot{x}^{2}+\dot{y}^{2}\right)+\frac{1}{2} m\left(x \dot{\theta}_{x}+y \dot{\theta}_{y}\right)^{2}+\frac{1}{2} I_{c d m}\left(\frac{\dot{x}^{2}+\dot{y}^{2}}{R}\right) \\
& +\frac{1}{2} I_{c d m}\left(\dot{\theta}_{x}^{2}+\dot{\theta}_{y}^{2}\right)-m G\left(x \sin \left(\theta_{x}\right)+y \sin \left(\theta_{y}\right)\right) .
\end{aligned}
$$

Lagrange's equations of motion are

$$
\begin{gathered}
\frac{\partial}{\partial t}\left[\frac{\partial L}{\partial \dot{x}}\right]-\frac{\partial L}{\partial x}=0, \\
\frac{\partial}{\partial t}\left[\frac{\partial L}{\partial \dot{\theta}_{x}}\right]-\frac{\partial L}{\partial \theta_{x}}=\tau_{x} \\
\frac{\partial}{\partial t}\left[\frac{\partial L}{\partial \dot{y}}\right]-\frac{\partial L}{\partial y}=0 \\
\frac{\partial}{\partial t}\left[\frac{\partial L}{\partial \dot{\theta}_{y}}\right]-\frac{\partial L}{\partial \theta_{y}}=\tau_{y}
\end{gathered}
$$




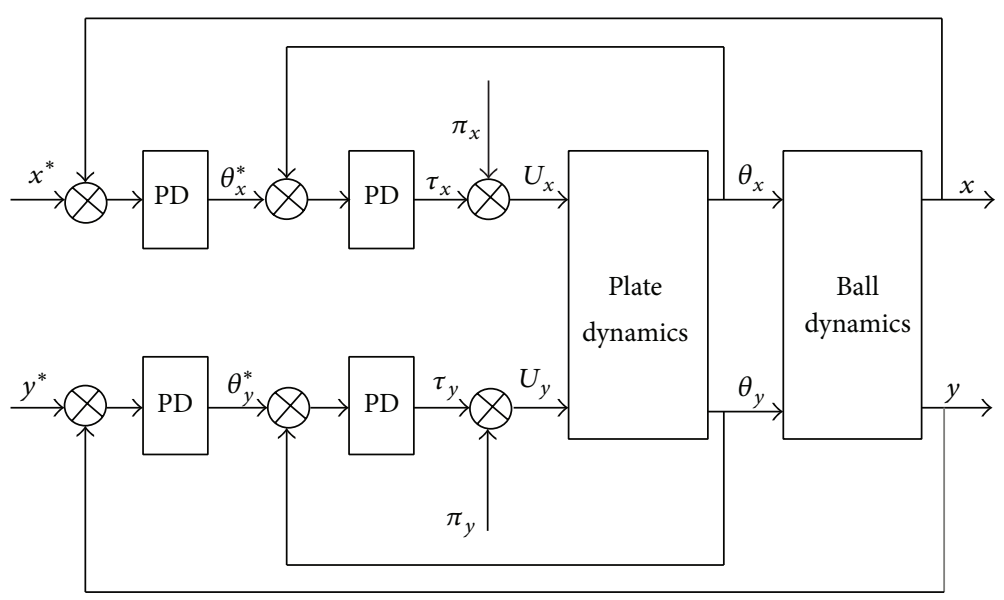

FIGURE 2: Control scheme of the ball and plate system.

where $\tau_{x}$ and $\tau_{y}$ are the torque applied to the plate. Developing (4) gives the whole ball and plate system as follows:

$$
\begin{gathered}
\left(m+\frac{I_{c d m}}{R^{2}}\right) \ddot{x}-m x \dot{\theta}_{x}^{2}-m y \dot{\theta}_{x} \dot{\theta}_{y}+m G \sin \theta_{x}=0 \\
\left(I_{c d m}+m x^{2}\right) \ddot{\theta}_{x}+2 m x \dot{x} \dot{\theta}_{x}+m x y \ddot{\theta}_{y} \\
+(m x \dot{y}+m \dot{x} y) \dot{\theta}_{y}+m G x \cos \theta_{x}=\tau_{x} \\
\left(m+\frac{I_{c d m}}{R^{2}}\right) \ddot{y}-m y \dot{\theta}_{y}^{2}-m x \dot{\theta}_{x} \dot{\theta}_{y}+m G \sin \theta_{y}=0 \\
\left(I_{c d m}+m y^{2}\right) \ddot{\theta}_{y}+2 m y \dot{y}_{y}+m x y \ddot{\theta}_{x} \\
+(m x \dot{y}+m \dot{x} y) \dot{\theta}_{x}+m G y \cos \theta_{y}=\tau_{y} .
\end{gathered}
$$

Define the system state as follows:

$$
q=\left[\begin{array}{llll}
x & \theta_{x} & y & \theta_{y}
\end{array}\right]^{T} .
$$

Define the regulation errors as follows:

$$
\bar{x}=x^{*}-x, \quad \bar{y}=y^{*}-y,
$$

where $x^{*}$ and $y^{*}$ are the desired ball position. For the ball and plate system, in the balance position $\theta_{x}^{*}=0, \theta_{y}^{*}=0, \dot{x}^{*}=0$, and $\dot{y}^{*}=0$, then $q^{*}=\left[\begin{array}{llll}x^{*} & 0 & y^{*} & 0\end{array}\right]^{T}$.

It is difficult to analyze the stability of the ball and plate system (5) with normal PD control. Nevertheless, it is well known that PD control can stabilize the manipulator robots [21]. Now, a transformation of the ball and plate closed-loop system will be discussed.

Lemma 1. The ball and plate closed-loop system (5) can be transformed into the following form:

$$
M(q) \ddot{q}+C(q, \dot{q}) \dot{q}+G(q)=B \bar{q}+D \pi,
$$

where $\bar{q}=q^{*}-q$, $q$ is defined in (6), and $q^{*}$ is defined in (7).

Proof. See Appendix A for the proof.

\section{Dual PD Control}

The goal of this research is to develop a PD regulation with compensation capable of controlling the position of ball on a plate in both axes $(x, y)$. Initially, the plate is at the horizontal, that is, $\theta_{x}=\theta_{y}=0$, and tilts in both axes to control the position of the ball. Each tilting axis will be operated by a DC motor. The position of the ball on the plate is measured by a camera [18].

A dual PD controller is an adequate basic term to obtain an acceptable response of the system; however, an exact nonlinear compensator is included to find the objective of guaranteeing the closed-loop system stability. Figure 2 shows a diagram of this controller scheme.

The dual PD control law of the $x$-axis is as follows:

$$
\begin{gathered}
U_{x}=k_{p m x}\left(\theta_{x}^{*}-\theta_{x}\right)+k_{d m x}\left(\dot{\theta}_{x}^{*}-\dot{\theta}_{x}\right)+\pi_{x}, \\
\theta_{x}^{*}=k_{p e x}\left(x^{*}-x\right)+k_{d e x}\left(\dot{x}^{*}-\dot{x}\right) .
\end{gathered}
$$

The dual PD control law of the $y$-axis is as follows:

$$
\begin{gathered}
U_{y}=k_{p m y}\left(\theta_{y}^{*}-\theta_{y}\right)+k_{d m y}\left(\dot{\theta}_{y}^{*}-\dot{\theta}_{y}\right)+\pi_{y}, \\
\theta_{y}^{*}=k_{p e y}\left(y^{*}-y\right)+k_{d e y}\left(\dot{y}^{*}-\dot{y}\right),
\end{gathered}
$$

where $k_{p m x}, k_{d m x}$ and $k_{p m y}, k_{d m y}$ are positive constants of the controllers of the plate and $k_{p e x}, k_{\text {dex }}$ and $k_{\text {pey }}$, $k_{d e y}$ are constants of controllers of the ball. $\pi_{x}$ and $\pi_{y}$ are compensators, which will be designed in the next section.

For the regulation problem, the control goal is to stabilize the ball in the desired position $\left(\dot{x}^{*}, \dot{y}^{*}\right)$, being denoted by $\left(\dot{x}^{*}, \dot{y}^{*}\right)=(0,0)$; therefore, the dual PD controllers become

$$
\begin{aligned}
U_{x}= & k_{p m x}\left[\left(k_{p e x}\left(x^{*}-x\right)+k_{d e x}\left(\dot{x}^{*}-\dot{x}\right)\right)-\theta_{x}\right] \\
& +k_{d m x}\left(\dot{\theta}_{x}^{*}-\dot{\theta}_{x}\right)+\pi_{x}, \\
U_{y}= & k_{p m y}\left[\left(k_{p e y}\left(y^{*}-y\right)+k_{d e y}\left(\dot{y}^{*}-\dot{y}\right)\right)-\theta_{y}\right] \\
& +k_{d m y}\left(\dot{\theta}_{y}^{*}-\dot{\theta}_{y}\right)+\pi_{y}
\end{aligned}
$$


or

$$
\begin{aligned}
U_{x}= & k_{p m x} k_{p e x} \bar{x}-\left(k_{p m x}+k_{d m x}\right) k_{d e x} \dot{x}-k_{d m x} k_{d e x} \ddot{x} \\
& -k_{p m x} \theta_{x}-k_{d m x} \dot{\theta}_{x}+\pi_{x}, \\
U_{y}= & k_{p m y} k_{p e y} \bar{y}-\left(k_{p m y}+k_{d m y}\right) k_{d e y} \dot{y}-k_{d m y} k_{d e y} \ddot{y} \\
& -k_{p m y} \theta_{y}-k_{d m y} \dot{\theta}_{y}+\pi_{y} .
\end{aligned}
$$

If $a_{1}=k_{\text {pmx }} k_{\text {pex }}, a_{2}=\left(k_{p m x}+k_{k d m x}\right) k_{\text {dex }}, a_{3}=k_{d m x} k_{d e x}$, $a_{4}=k_{p m x}, a_{5}=k_{d m x}, b_{1}=k_{p m y} k_{p e y}, b_{2}=\left(k_{p m y}+k_{k d m y}\right) k_{d e y}$, $b_{3}=k_{d m y} k_{d e y}, b_{4}=k_{p m y}$, and $b_{5}=k_{d m y}, U_{x}$ and $U_{y}$ can be written as follows:

$$
\begin{aligned}
& U_{x}=-a_{1} \bar{x}-a_{2} \dot{x}-a_{3} \ddot{x}-a_{4} \theta_{x}-a_{5} \dot{\theta}_{x}+\pi_{x}, \\
& U_{y}=-b_{1} \bar{y}-b_{2} \dot{y}-b_{3} \ddot{y}-b_{4} \theta_{y}-b_{5} \dot{\theta}_{y}+\pi_{y} .
\end{aligned}
$$

\section{Stability Analysis}

In this section, Lyapunov's first method is used to prove the dual PD control with nonlinear compensation (14) and (15) is asymptotically stable.

First, it is analyzed if the matrices $M$ and $B$ may be candidates for Lyapunov function. The matrix $M$ is not symmetric to prove that the function is positive; that is, each main minor determinate is computed. If the constants $m>0$, $R>0, I_{c d m}>0$, and $M$ is a square matrix, then

$$
\operatorname{Det}(1,1)=m+\frac{1}{R^{2}} I_{c d m}>0
$$

$\operatorname{Det}(2,2)=\frac{1}{R^{2}}\left(R^{2} m^{2} x^{2}+R^{2} m I_{c d m}+m x^{2} I_{c d m}+I_{c d m}^{2}\right)>0$,

$$
\begin{aligned}
\operatorname{Det}(3,3)=\frac{1}{R^{4}} & \left(R^{4} m^{3} x^{2}+R^{4} m^{2} I_{c d m}+2 R^{2} m^{2} x^{2} I_{c d m}\right. \\
& \left.+2 R^{2} m I_{c d m}^{2}+m x^{2} I_{c d m}^{2}+I_{c d m}^{3}\right)>0,
\end{aligned}
$$

$\operatorname{Det}(4,4)=\frac{1}{R^{4}}\left(R^{4} m^{3} x^{2} I_{c d m}+R^{4} m^{3} y^{2} I_{c d m}+R^{4} m^{2} I_{c d m}^{2}\right.$

$$
\begin{aligned}
& +2 R^{2} m^{2} x^{2} I_{c d m}^{2}+2 R^{2} m^{2} y^{2} I_{c d m}^{2} \\
& +2 R^{2} m I_{c d m}^{3}+m x^{2} I_{c d m}^{3} \\
& \left.+m y^{2} I_{c d m}^{3}+I_{c d m}^{4}\right)>0 .
\end{aligned}
$$

Position of the ball is $(x, y)>0$; therefore, the matrix $M$ that satisfies all determinants of the main minors (16) are positive; then, $M$ is a positive definite matrix that satisfies

$$
\begin{aligned}
& V(0)=\dot{q}^{T} M(q) \dot{q}=0, \\
& V(q)=\dot{q}^{T} M(q) \dot{q}>0 .
\end{aligned}
$$

The matrix $B$ is not symmetric to prove that the function is positive and the calculation of quadratic form is determined. Consider

$$
\bar{q}^{T} B \bar{q}=a_{4} \theta_{x}^{2}+x a_{1} \theta_{x}+b_{4} \theta_{y}^{2}+y b_{1} \theta_{y} .
$$

The position of the plate is $\left(\theta_{x}, \theta_{y}\right)>0$, and the constants are $a_{4}>0, a_{1}>0, b_{4}>0, b_{1}>0$. If $B$ is a square matrix (A.5), then the quadratic form satisfies

$$
\begin{aligned}
& V(0)=\bar{q}^{T} B \bar{q}=0, \\
& V(q)=\bar{q}^{T} B \bar{q}>0 .
\end{aligned}
$$

The stability of the closed-loop system is stated in the following theorem.

Theorem 2. Consider the ball and plate system (8), the dual PD control (14), (15), and the following compensators of $\pi_{x}$ and $\pi_{y}$ as follows

$$
\begin{aligned}
& \pi_{x}= \begin{cases}a_{2} \dot{x}-m x \dot{\theta}_{x}+m x \dot{x} \dot{\theta}_{x}-m x \dot{\theta}_{y}+m y \dot{x} \dot{\theta}_{y} & \text { if } \dot{x} \neq 0 \\
-m x \dot{\theta}_{x}-m x \dot{\theta}_{y} & \text { if } \dot{x}=0\end{cases} \\
& \pi_{y}= \begin{cases}b_{2} \dot{y}-m y \dot{\theta}_{y}+m y \dot{y}_{y}-m y \dot{\theta}_{x}+m x \dot{y} \dot{\theta}_{x} & \text { if } \dot{y} \neq 0 \\
-m y \dot{\theta}_{y}-m y \dot{\theta}_{x} & \text { if } \dot{y}=0 .\end{cases}
\end{aligned}
$$

Therefore, the closed-loop system of the ball and plate system is asymptotically stable

$$
\begin{aligned}
& \lim _{t \rightarrow \infty} \bar{x}(t)=0, \\
& \lim _{t \rightarrow \infty} \bar{y}(t)=0 .
\end{aligned}
$$

Proof. Since $M(x)$ is a positive definite matrix, $B$ in (18) is a quadratic form, and recalling that $\theta_{x}$ and $\theta_{y}$ are not negative, the following positive definite quadratic form is used as the Lyapunov function:

$V(q, \dot{q})=\frac{1}{2} \dot{q}^{T} M(q) \dot{q}+\frac{1}{2} \bar{q}^{T} B \bar{q}+m G x \sin \theta_{x}+m G y \sin \theta_{y}$.

To assure the potential energy $E_{p}=m G\left(x \sin \left(\theta_{x}\right)+y \sin \left(\theta_{y}\right)\right)$ is positive, we let $\theta_{x} \geq 0$ and $\theta_{y} \geq 0, V(x, \dot{x}) \geq 0$. Differentiating it with respect to time and recalling that $x^{*}$ and $y^{*}$ are constants, it yields

$$
\begin{aligned}
d V= & \dot{q}^{T} M(q) \ddot{q}+\frac{1}{2} \dot{q}^{T} \dot{M}(q) \dot{q}-\dot{q}^{T} B \bar{q}+m G \dot{x} \sin \theta_{x} \\
& +m G x \cos \theta_{x} \dot{\theta}_{x}+m G \dot{y} \sin \theta_{y}+m G y \cos \theta_{y} \dot{\theta}_{y} .
\end{aligned}
$$

It is (see Appendix B)

$$
\begin{aligned}
d V= & -a_{5} \dot{\theta}_{x}^{2}+\dot{\theta}_{x}\left[\pi_{x}-a_{2} \dot{x}+m x \dot{\theta}_{x}-m x \dot{x}_{x}\right] \\
& +\dot{\theta}_{x}\left[+m x \dot{\theta}_{y}-m y \dot{x} \dot{\theta}_{y}-m G x \cos \theta_{x}+m G x \cos \theta_{x}\right] \\
& -m \dot{x} G \sin \theta_{x}+m G \dot{x} \sin \theta_{x}-b_{5} \dot{\theta}_{y}^{2} \\
& +\dot{\theta}_{y}\left[\pi_{y}-b_{2} \dot{y}+m y \dot{\theta}_{y}-m y \dot{y} \dot{\theta}_{y}+m y \dot{\theta}_{x}-m x \dot{y}\right] \\
& +\dot{\theta}_{y}\left[-m G y \cos \theta_{y}+m G y \cos \theta_{y}\right] \\
& -m \dot{y} G \sin \theta_{y}+m G \dot{y} \sin \left(\theta_{y}\right) .
\end{aligned}
$$


Using the compensators (20) in (24) gives

$$
d V \leq-a_{5} \dot{\theta}_{x}^{2}-b_{5} \dot{\theta}_{y}^{2}
$$

Since $a_{5}>0$ and $b_{5}>0, d V$ is a semidefinite negative function. Thus, the closed-loop system is stable in the equilibrium point $[\dot{x}, \dot{y}]=[0,0]$. To prove asymptotic stability, LaSalle's theorem is used in the following region:

$$
\Omega=\left\{\left[x, \theta_{x}, y, \theta_{y}\right] \mid d V=0\right\} .
$$

From (22), $d V=0$ if and only if $\dot{\theta}_{x}=\dot{\theta}_{y}=0$. For a solution $\theta_{x, y}(t)$ to belong to $\Omega$ for all $t \geq 0$, it is necessary and sufficient that $\theta_{x}=\theta_{y}=0$ for all $t \geq 0$. Therefore it must also hold that $x=y=0$ for all $t \geq 0$. It is concluded that the closed-loop system is the only initial condition in $\Omega$ for which $x(t) \in \Omega$ for all $t \geq 0$. Finally, the origin of the closed-loop system is asymptotically stable in $\left[x, \theta_{x}, y, \theta_{y}\right]=[0,0,0,0][6-10]$.

Remark 3. In [22-26], the authors use proportional-integralderivative controllers; nevertheless, the proposed controller is different because it has 4 dynamic alternatives being selected by the controller depending on the $x$ and $y$ values of the ball and beam system, and it is called the dual PD control. The proposed controller has the main merit that it can guarantee the convergence of the regulation error; that is, the dual PD controller obtains a desired behavior in the system. The shortcoming of the proposed method is that the changes between the 4 dynamic alternatives in the controller could cause the undesired chattering.

\section{Prototype}

Figure 3 shows the prototype used to test the new dual PD controller. Simulink is used to test the model (8); consider $M=0.11 \mathrm{~kg}, R=1.27 \mathrm{~cm}$, and $G=9.81 \mathrm{~m} / \mathrm{s}^{2}$. Once the simulation is successful, the implementation on FPGA is carried out. The prototype has the following parts: the plate size is $50 \times 50 \mathrm{~cm}$, the ball is with a radius of $1.27 \mathrm{~cm}$, and a CMOS camera model MT9 M011 [27] is used as a visual position sensor of the ball. The model used as image sensor is the TRDB card DC2 [27] of Terasic company. The designed hardware components of the prototype are image preprocessing, ball position, and dual $\mathrm{PD}$ controller with compensator. The image preprocessing component receives a color image from CMOS camera and delivers a binary image. The ball position component receives the binary image and delivers the position $(x, y)$ of the ball on the plate; these components are able to process 16 images per second [18]; the position of the ball is sent to the dual PD controller component; this includes the new control law. Finally, the motors receive these control laws and they move the plate on $x$ and $y$ directions, simultaneously. The DC motors are selected as an actuator for its high torque. This motor is a powerful $12 \mathrm{~V}$ brushed DC motor with a 50:1 metal gearbox and an integrated quadrature encoder [28]. To obtain realtime operation, all modules are embedded in hardware using the very high speed hardware description language (VHDL) using Quartus II version 7.2 and are implemented in Altera
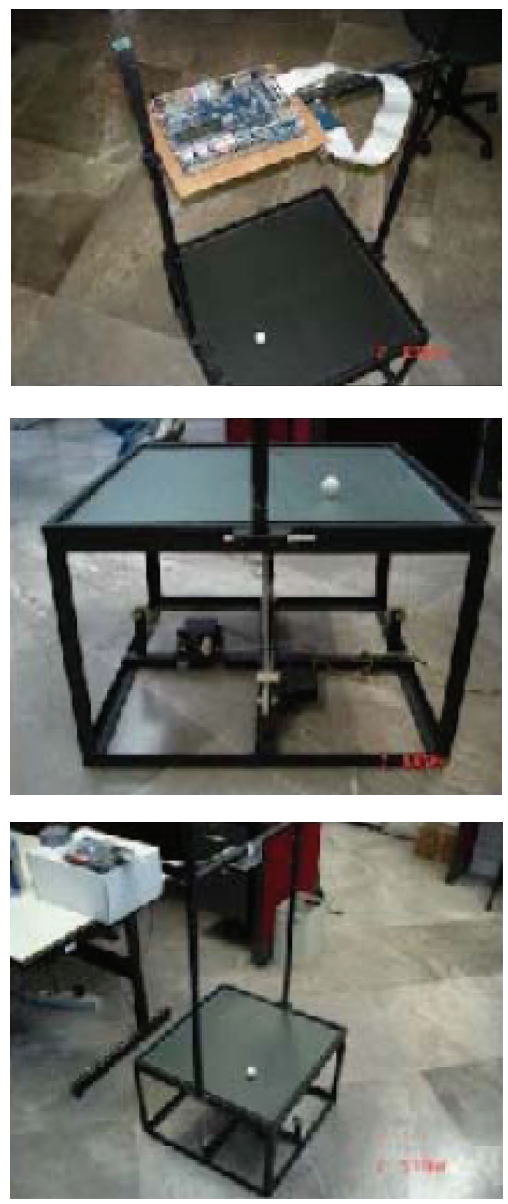

FIGURE 3: Ball and plate prototype.

DE2 [29] (Development Education board) with a Cyclone II FPGA.

\section{Experimental Results}

To verify the advantages of the dual PD controller with compensator, several experiments are performed for the regulation [11], [12]. A first set of experiments are performed using the proposed method which implements the control laws (13) and (14); these experiments are repeated using the dual PD controller without compensator, meaning $\pi_{x}=\pi_{y}=$ 0 in (13) and (14). Finally, the proposed controller is compared with a fuzzy logic controller [18] using the same experiments.

Consider that the movement of the ball is slow and does not show tendency to slip (smooth bearing), due to the slow speed and acceleration of the plate; the interactions of the movements of the plate are uncoupled; the details of the performance are shown in Figures 4-6, where the position, velocity, and control signals are presented as the dual PD control with compensator, PD control without compensation, and fuzzy logic controller responses.

Figure 4 shows the position of the ball on the $x$-axis; in this experiment the initial position is $0.02587 \mathrm{~m}$ and the final position is $0.4375 \mathrm{~m}$, and it shows that the dual PD control 


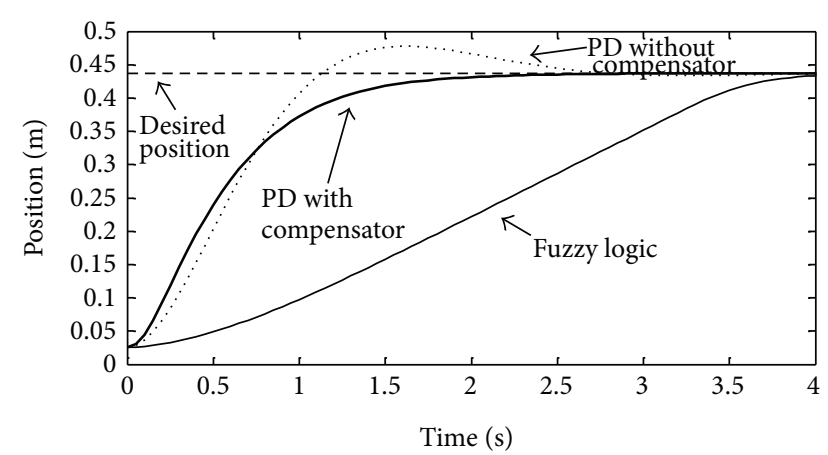

Figure 4: Position of the ball on the $x$-axis.

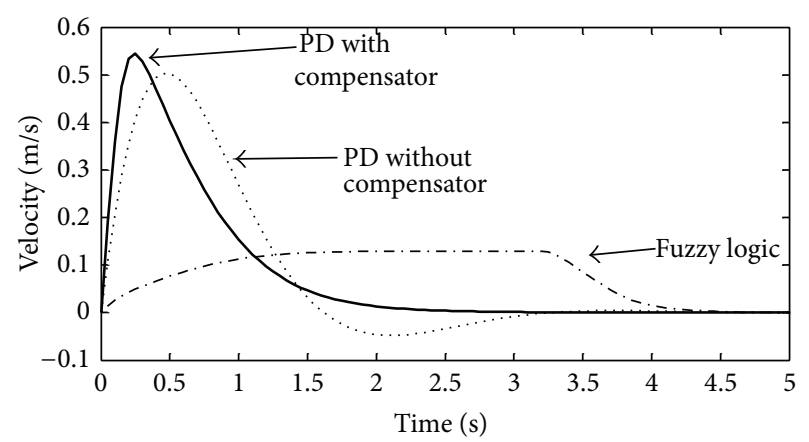

FIGURE 5: Velocity of the ball on the $x$-axis.

with compensator reaches the desired value faster than the other two methods. Figure 5 shows behavior of the velocity of the ball on the $x$-axis; in this experiment the initial velocity is 0 , and it shows that the dual PD control with compensator is stabilized faster than the other methods. Figure 6 shows behavior of the control signals on the $x$-axis, where the dual PD control with compensator shows the best behavior.

\section{Conclusions and Future Work}

The dual PD control with nonlinear compensation has been presented to solve the regulation problem for the ball and plate system. To use these controllers, a new dynamic model in the manipulator robot form was obtained. The proposed nonlinear model is very useful to design and validate different control algorithms which can then be extrapolated to problems with the same characteristics; one advantage of working with a nonlinear model is that the full dynamic can be seen, making it possible to analyze the behavior of the system at each equilibrium point. By using the first method of Lyapunov, a new Lyapunov function is presented for the stability analysis; as a result of this analysis, the asymptotic stability of the closed-loop system can be guaranteed. On the order hand, the exact compensator requires to have a precise knowledge of the system nonlinearities; however, the methodology of how to obtain it is presented in detail. Furthermore, the experimental result shows the excellent performance of the proposed controller, obtaining the best behavior in comparison with the other selected controllers;

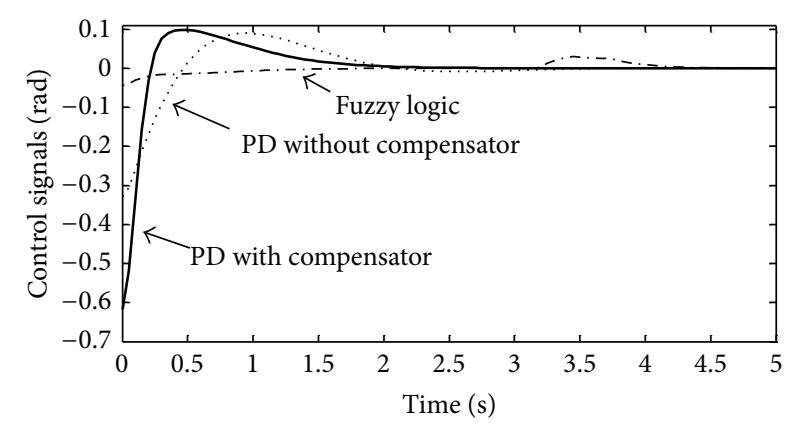

Figure 6: Control of the ball on the $x$-axis.

see Figures 4, 5, and 6. As a future work, a PD regulator with an intelligent compensator will be developed [30-33].

\section{Appendices}

\section{A. Ball and Plate Model}

Substituting (14) and (15) into (5), we have

$$
\begin{gathered}
\left(m+\frac{I_{c d m}}{R^{2}}\right) \ddot{x}-m x \dot{\theta}_{x}^{2}-m y \dot{\theta}_{x} \dot{\theta}_{y}+m G \sin \theta_{x}=0, \\
\left(I_{c d m}+m x^{2}\right) \ddot{\theta}_{x}+2 m x \dot{x}_{x}+m x y \ddot{\theta}_{y} \\
+(m x \dot{y}+m \dot{x} y) \dot{\theta}_{y}+m G x \cos \theta_{x}=U_{x}, \\
\left(m+\frac{I_{c d m}}{R^{2}}\right) \ddot{y}-m y \dot{\theta}_{y}^{2}-m x \dot{\theta}_{x} \dot{\theta}_{y}+m G \sin \theta_{y}=0, \\
\left(I_{c d m}+m y^{2}\right) \ddot{\theta}_{y}+2 m y \dot{y}_{y}+m x y \ddot{\theta}_{x} \\
+(m x \dot{y}+m \dot{x} y) \dot{\theta}_{x}+m G y \cos \theta_{y}=U_{y} .
\end{gathered}
$$

By equating all the equations to zero, we have

$$
\begin{aligned}
& \left(m+\frac{I_{c d m}}{R^{2}}\right) \ddot{x}-m x \dot{\theta}_{x}^{2}-m y \dot{\theta}_{x} \dot{\theta}_{y}+m G \sin \theta_{x}=0, \\
& \left(I_{c d m}+m x^{2}\right) \ddot{\theta}_{x}+2 m x \dot{x}_{x}+m x y \ddot{\theta}_{y} \\
& \quad+(m x \dot{y}+m \dot{x} y) \dot{\theta}_{y}+m G x \cos \theta_{x}+a_{1} \bar{x}+a_{2} \dot{x} \\
& \quad+a_{3} \ddot{x}+a_{4} \theta_{x}+a_{5} \dot{\theta}_{x}-\pi_{x}=0, \\
& \left(m+\frac{I_{c d m}}{R^{2}}\right) \ddot{y}-m y \dot{\theta}_{y}^{2}-m x \dot{\theta}_{x} \dot{\theta}_{y}+m G \sin \theta_{y}=0, \\
& \left(I_{c d m}+m y^{2}\right) \ddot{\theta}_{y}+2 m y \dot{y} \dot{\theta}_{y}+m x y \ddot{\theta}_{x} \\
& \quad+(m x \dot{y}+m \dot{x} y) \dot{\theta}_{x}+m G y \cos \theta_{y}+b_{1} \bar{y}+b_{2} \dot{y} \\
& \quad+b_{3} \ddot{y}+b_{4} \theta_{y}+b_{5} \dot{\theta}_{y}-\pi_{y}=0 .
\end{aligned}
$$


The inertia matrix $M(q)$ is

$$
M(q)=\left[\begin{array}{cccc}
l_{1} & 0 & 0 & 0 \\
a_{3} & I_{c d m}+m x^{2} & 0 & m x y \\
0 & 0 & l_{2} & 0 \\
0 & m x y & b_{3} & I_{c d m}+m y^{2}
\end{array}\right]
$$

where $l_{1}=m+\left(I_{c d m} / R^{2}\right)$ and $l_{2}=m+\left(I_{c d m} / R^{2}\right)$. The Coriolis matrix $C(q, \dot{q})$ is

$$
C(q, \dot{q})=\left[\begin{array}{cccc}
0 & -m x \dot{\theta}_{x} & 0 & -m y \dot{\theta}_{x} \\
l_{3} & a_{5} & 0 & m x \dot{y}+m \dot{x} y \\
0 & -m x \dot{\theta}_{y} & 0 & -m y \dot{\theta}_{y} \\
0 & m x \dot{y}+m \dot{x} y & l_{4} & b_{5}
\end{array}\right],
$$

where $l_{3}=2 m x \dot{\theta}_{x}+a_{2}$ and $l_{4}=2 m y \dot{\theta}_{y}+b_{2}$. The matrix $B$ is

$$
B=\left[\begin{array}{cccc}
0 & 0 & 0 & 0 \\
a_{1} & a_{4} & 0 & 0 \\
0 & 0 & 0 & 0 \\
0 & 0 & b_{1} & b_{4}
\end{array}\right]
$$

The gravity matrix $G$ is

$$
G(q)=\left[\begin{array}{c}
m G \sin \theta_{x} \\
m G x \cos \theta_{x} \\
m G \sin \theta_{y} \\
m G y \cos \theta_{y}
\end{array}\right]
$$

The compensator matrix $D \pi$ is

$$
D \pi=\left[\begin{array}{c}
0 \\
\pi_{x} \\
0 \\
\pi_{y}
\end{array}\right]
$$

Therefore the ball and plate system model can be described in the form (8).

\section{B. Stability Analysis}

From (8), the inertial matrix $M(q) \ddot{q}$ is

$$
M(q) \ddot{q}=B \bar{q}+D \pi-C(q, \dot{q}) \dot{q}-G(q) .
$$

Substituting (B.1) into (23), we have

$$
\begin{aligned}
d V= & \dot{q}^{T}[B \bar{q}+D \pi-C(q, \dot{q}) \dot{q}-G(q)] \\
& +\frac{1}{2} \dot{q}^{T} \dot{M}(q) \dot{q}-\dot{q}^{T} B \bar{q}+m G \dot{x} \sin \theta_{x} \\
& +m G x \cos \theta_{x} \dot{\theta}_{x}+m G \dot{y} \sin \theta_{y}+m G y \cos \theta_{y} \dot{\theta}_{y} .
\end{aligned}
$$

Developing (B.2), we have

$$
\begin{aligned}
d V= & \dot{q}^{T} B \bar{q}+\dot{q}^{T} D \pi-\dot{q}^{T} C(q, \dot{q}) \dot{q}-\dot{q}^{T} G(q) \\
& +\frac{1}{2} \dot{q}^{T} \dot{M}(q) \dot{q}-\dot{q}^{T} B \bar{q}+m G \dot{x} \sin \theta_{x} \\
& +m G x \cos \theta_{x} \dot{\theta}_{x}+m G \dot{y} \sin \theta_{y}+m G y \cos \theta_{y} \dot{\theta}_{y} .
\end{aligned}
$$

Factoring similar terms, we have

$$
\begin{aligned}
d V= & \dot{q}^{T}[B \bar{q}-B \bar{q}+D \pi-G(q)] \\
& +\frac{1}{2} \dot{q}^{T} \dot{M}(q) \dot{q}-\dot{q}^{T} C(q, \dot{q}) \dot{q}+m G \dot{x} \sin \theta_{x} \\
& +m G x \cos \theta_{x} \dot{\theta}_{x}+m G \dot{y} \sin \theta_{y}+m G y \cos \theta_{y} \dot{\theta}_{y} .
\end{aligned}
$$

Eliminating and grouping terms, we have

$$
\begin{aligned}
d V= & \dot{q}^{T}[D \pi-G(q)]+\frac{1}{2} \dot{q}^{T}[\dot{M}(q)-2 C(q, \dot{q})] \dot{q} \\
& +m G \dot{x} \sin \theta_{x}+m G x \cos \theta_{x} \dot{\theta}_{x} \\
& +m G \dot{y} \sin \theta_{y}+m G y \cos \theta_{y} \dot{\theta}_{y} .
\end{aligned}
$$

From (B.5), the differentiate of $M(q)$ is

$$
\dot{M}=\left[\begin{array}{cccc}
0 & 0 & 0 & 0 \\
0 & 2 m x & 0 & m(x+y) \\
0 & 0 & 0 & 0 \\
0 & m(x+y) & 0 & 2 m y
\end{array}\right] .
$$

Equation (B.6) is substituted in $[\dot{M}(q)-2 C(q, \dot{q})]$ as follows:

$$
\begin{aligned}
& {[\dot{M}(q)-2 C(q, \dot{q})]} \\
& =\left[\begin{array}{cccc}
0 & 0 & 0 & 0 \\
0 & 2 m x & 0 & m(x+y) \\
0 & 0 & 0 & 0 \\
0 & m(x+y) & 0 & 2 m y
\end{array}\right]-\cdots \\
& -2\left[\begin{array}{cccc}
0 & -m x \dot{\theta}_{x} & 0 & -m y \dot{\theta}_{x} \\
l_{5} & a_{5} \dot{\theta}_{y} & 0 & m x \dot{y}+m \dot{x} y \\
0 & -m x \dot{\theta}_{y} & 0 & -m y \dot{\theta}_{y} \\
0 & m x \dot{y}+m \dot{x} y & l_{6} & b_{5}
\end{array}\right],
\end{aligned}
$$

where $l_{5}=2 m x \dot{\theta}_{x}+a_{2}$ and $l_{6}=2 m y \dot{\theta}_{y}+b_{2}$. Developing (B.7) and separating terms variables constants, we have

$$
\begin{aligned}
& {\left[\begin{array}{cccc}
0 & 2 m x \dot{\theta}_{x} & 0 & 2 m y \dot{\theta}_{x} \\
-4 m x \dot{\theta}_{x} & 2 m x & 0 & l_{8} \dot{\theta}_{y} \\
0 & 2 m x \dot{\theta}_{y} & 0 & 2 m y \dot{\theta}_{y} \\
0 & l_{7} & -4 m y \dot{\theta}_{y} & 2 m y
\end{array}\right]} \\
& -\left[\begin{array}{cccc}
0 & 0 & 0 & 0 \\
2 a_{2} & 2 a_{5} & 0 & 0 \\
0 & 0 & 0 & 0 \\
0 & 0 & 2 b_{2} & 2 b_{5}
\end{array}\right],
\end{aligned}
$$

where $l_{7}=m(x+y)-2 m x \dot{y}-2 m \dot{x} y$ and $l_{8}=-2 m x \dot{y}-$ $2 m \dot{x} y+m(x+y)$. We define a new matrix $P$ :

$$
P=\left[\begin{array}{cccc}
0 & 0 & 0 & 0 \\
-2 a_{2} & -2 a_{5} & 0 & 0 \\
0 & 0 & 0 & 0 \\
0 & 0 & -2 b_{2} & -2 b_{5}
\end{array}\right]
$$


Differentiating (6), we have

$$
\dot{q}=\left[\begin{array}{c}
\dot{x} \\
\dot{\theta}_{x} \\
\dot{y} \\
\dot{\theta}_{y}
\end{array}\right] \dot{q}^{T}=\left[\begin{array}{llll}
\dot{x} & \dot{\theta}_{x} & \dot{y} & \dot{\theta}_{y}
\end{array}\right]
$$

$(1 / 2) \dot{q}^{T} P \dot{q}$ is calculated by multiplying $\dot{q}^{T} P$ :

$$
\begin{gathered}
\dot{q}^{T} P=\left[\begin{array}{llll}
\dot{x} & \dot{\theta}_{x} & \dot{y} & \dot{\theta}_{y}
\end{array}\right]\left[\begin{array}{cccc}
0 & 0 & 0 & 0 \\
-2 a_{2} & -2 a_{5} & 0 & 0 \\
0 & 0 & 0 & 0 \\
0 & 0 & -2 b_{2} & -2 b_{5}
\end{array}\right], \\
\dot{q}^{T} P=\left[\begin{array}{llll}
-2 a_{2} \dot{\theta}_{x} & -2 a_{5} \dot{\theta}_{x} & -2 b_{2} \dot{\theta}_{y} & -2 b_{5} \dot{\theta}_{y}
\end{array}\right] .
\end{gathered}
$$

After multiplying (B.12) by $\dot{q}$, we have

$$
\begin{gathered}
\dot{q}^{T} P \dot{q}=\left[\begin{array}{llll}
-2 a_{2} \dot{\theta}_{x} & -2 a_{5} \dot{\theta}_{x} & -2 b_{2} \dot{\theta}_{y} & -2 b_{5} \dot{\theta}_{y}
\end{array}\right]\left[\begin{array}{c}
\dot{x} \\
\dot{\theta}_{x} \\
\dot{y} \\
\dot{\theta}_{y}
\end{array}\right], \\
\dot{q}^{T} P \dot{q}=-2 a_{2} \dot{\theta}_{x} \dot{x}-2 a_{5} \dot{\theta}_{x}^{2}-2 b_{2} \dot{\theta}_{y} \dot{y}-2 b_{5} \dot{\theta}_{y}^{2} .
\end{gathered}
$$

Finally multiplying (B.14) by (1/2),we have

$$
\frac{1}{2} \dot{q}^{T} P \dot{q}=-a_{2} \dot{\theta}_{x} \dot{x}-a_{5} \dot{\theta}_{x}^{2}-b_{2} \dot{\theta}_{y} \dot{y}-b_{5} \dot{\theta}_{y}^{2}
$$

Equation (B.8) is calculated as follows:

$$
\frac{1}{2} \dot{q}^{T}\left[\begin{array}{cccc}
0 & 2 m x \dot{\theta}_{x} & 0 & 2 m y \dot{\theta}_{x} \\
-4 m x \dot{\theta}_{x} & 2 m x & 0 & l_{8} \\
0 & 2 m x \dot{\theta}_{y} & 0 & 2 m y \dot{\theta}_{y} \\
0 & l_{7} & -4 m y \dot{\theta}_{y} & 2 m y
\end{array}\right] \dot{q},
$$

where $l_{7}$ and $l_{8}$ are given in (B.8). From (B.16), we first have

$$
\begin{aligned}
& {\left[\begin{array}{llll}
\dot{x} & \dot{\theta}_{x} & \dot{y} & \dot{\theta}_{y}
\end{array}\right]\left[\begin{array}{cccc}
0 & 2 m x \dot{\theta}_{x} & 0 & 2 m y \dot{\theta}_{x} \\
-4 m x \dot{\theta}_{x} & 2 m x & 0 & l_{8} \\
0 & 2 m x \dot{\theta}_{y} & 0 & 2 m y \dot{\theta}_{y} \\
0 & l_{7} & -4 m y \dot{\theta}_{y} & 2 m y
\end{array}\right]} \\
& =\left[\begin{array}{llll}
-4 m x \dot{\theta}_{x}^{2} & l_{9} & -4 m y \dot{\theta}_{y}^{2} & l_{10}
\end{array}\right],
\end{aligned}
$$

where $l_{9}=2 m x \dot{x} \dot{\theta}_{x}+2 m x \dot{\theta}_{x}+2 m x \dot{y} \dot{\theta}_{y}+m \dot{\theta}_{y}(x+y)-$ $2 m \dot{\theta}_{y} x \dot{y}-2 m \dot{\theta}_{y} \dot{x} y, l_{10}=2 m y \dot{x} \dot{\theta}_{x}-2 m \dot{\theta}_{x} x \dot{y}-2 m \dot{\theta}_{x} \dot{x} y+$ $m \dot{\theta}_{x}(x+y)+2 m y \dot{y} \dot{\theta}_{y}+2 m y \dot{\theta}_{y}$, and $l_{7}$ and $l_{8}$ are given in (B.16). Then multiplying (B.17) by $\dot{q}$, we have

$$
\begin{aligned}
& {\left[\begin{array}{llll}
-4 m x \dot{\theta}_{x}^{2} & l_{9} & -4 m y \dot{\theta}_{y}^{2} & l_{10}
\end{array}\right]\left[\begin{array}{c}
\dot{x} \\
\dot{\theta}_{x} \\
\dot{y}_{\dot{\theta}_{y}}
\end{array}\right],} \\
& =-4 m x \dot{x} \dot{\theta}_{x}^{2}+2 m x \dot{x} \dot{\theta}_{x}^{2}+2 m x \dot{\theta}_{x}^{2}+2 m x \dot{y} \dot{\theta}_{y} \dot{\theta}_{x} \\
& +m \dot{\theta}_{y} \dot{\theta}_{x}(x+y)-2 m \dot{\theta}_{y} \dot{\theta}_{x} x \dot{y}-2 m \dot{\theta}_{y} \dot{\theta}_{x} \dot{x} y-\cdots \\
& -4 m y \dot{y} \dot{\theta}_{y}^{2}+2 m y \dot{x} \dot{\theta}_{x} \dot{\theta}_{y}-2 m \dot{\theta}_{x} \dot{\theta}_{y} x \dot{y}-2 m \dot{\theta}_{x} \dot{\theta}_{y} \dot{x} y \\
& +m \dot{\theta}_{x} \dot{\theta}_{y}(x+y)+2 m y \dot{y} \dot{\theta}_{y}^{2}+2 m y \dot{\theta}_{y}^{2} \text {, } \\
& =-2 m x \dot{x} \dot{\theta}_{x}^{2}-2 m y \dot{y} \dot{\theta}_{y}^{2}+2 m x \dot{\theta}_{x}^{2}+2 m y \dot{\theta}_{y}^{2} \\
& +2 m x \dot{\theta}_{y} \dot{\theta}_{x}+2 m y \dot{\theta}_{y} \dot{\theta}_{x}-2 m x \dot{y} \dot{\theta}_{y} \dot{\theta}_{x}-2 m \dot{x} y \dot{\theta}_{x} \dot{\theta}_{y} \text {. }
\end{aligned}
$$

Finally multiplying (B.20) by $1 / 2$, we obtain

$$
\begin{aligned}
= & -m x \dot{x} \dot{\theta}_{x}^{2}-m y \dot{y} \dot{\theta}_{y}^{2}+m x \dot{\theta}_{x}^{2}+m y \dot{\theta}_{y}^{2} \\
& +m x \dot{\theta}_{y} \dot{\theta}_{x}+m y \dot{\theta}_{y} \dot{\theta}_{x}-m x \dot{y}_{y} \dot{\theta}_{x}-m \dot{x} y \dot{\theta}_{x} \dot{\theta}_{y} .
\end{aligned}
$$

Therefore, the term $(1 / 2) \dot{q}^{T}[\dot{M}(q)-2 C(q, \dot{q})] \dot{q}$ is equal to the sum of (B.21) and (B.15) as follows:

$$
\begin{aligned}
= & -a_{2} \dot{\theta}_{x} \dot{x}-a_{5} \dot{\theta}_{x}^{2}-b_{2} \dot{\theta}_{y} \dot{y}-b_{5} \dot{\theta}_{y}^{2} \\
& -m x \dot{x} \dot{\theta}_{x}^{2}-m y \dot{y} \dot{\theta}_{y}^{2}+m x \dot{\theta}_{x}^{2}+m y \dot{\theta}_{y}^{2}+m x \dot{\theta}_{y} \dot{\theta}_{x} \\
& +m y \dot{\theta}_{y} \dot{\theta}_{x}-\cdots-m x \dot{y} \dot{\theta}_{y} \dot{\theta}_{x}-m \dot{x}_{y} \dot{\theta}_{x} \dot{\theta}_{y} .
\end{aligned}
$$

From (B.5), we calculate the term $-\dot{q}^{T} G(q)$ as follows:

$$
\begin{aligned}
= & -\left[\begin{array}{llll}
\dot{x} & \dot{\theta}_{x} & \dot{y} & \dot{\theta}_{y}
\end{array}\right]\left[\begin{array}{c}
m G \sin \theta_{x} \\
m G x \cos \theta_{x} \\
m G \sin \theta_{y} \\
m G y \cos \theta_{y}
\end{array}\right] \\
= & -m \dot{x} G \sin \theta_{x}-m x \dot{\theta}_{x} G \cos \theta_{x} \\
& -m \dot{y} G \sin \theta_{y}-m y \dot{\theta}_{y} G \cos \theta_{y} .
\end{aligned}
$$

Finally, the term is calculated $\dot{q}^{T} D \pi$ from (B.5):

$$
\left[\begin{array}{llll}
\dot{x} & \dot{\theta}_{x} & \dot{y} & \dot{\theta}_{y}
\end{array}\right]\left[\begin{array}{c}
0 \\
\pi_{x} \\
0 \\
\pi_{y}
\end{array}\right]=\pi_{x} \dot{\theta}_{x}+\pi_{y} \dot{\theta}_{y} .
$$

Therefore, to obtain the derivative of the Lyapunov function, add (B.22), (B.23), and (B.24), and factoring $\dot{\theta}_{x}$ and $\dot{\theta}_{y}$, we have (24). 


\section{Conflict of Interests}

The authors declare no conflict of interests about all the aspects related to this paper.

\section{Acknowledgments}

The authors wish to thank the anonymous reviewers for their detailed reviews and many constructive suggestions which have improved the paper significantly and to the Institutions of Mexico (SNI-CONACyT, SIP-IPN, COFAA-IPN, PIFIIPN, and CONACyT) for providing necessary support to carry out this research work.

\section{References}

[1] Y. Tian, M. Bai, and J. Su, "A non-linear switching controller for ball and plate system," International Journal of Modelling, Identification and Control, vol. 1, no. 3, pp. 177-182, 2006.

[2] S.-K. Oh, H.-J. Jang, and W. Pedrycz, "The design of a fuzzy cascade controller for ball and beam system: a study in optimization with the use of parallel genetic algorithms," Engineering Applications of Artificial Intelligence, vol. 22, no. 2, pp. 261271, 2009.

[3] Y. Liu and H. Yu, "Fuzzy control of a pendulum-driven cart," in Proceedings of the International Conference on Modelling, Identification and Control (ICMIC '10), pp. 698-703, Okayama, Japan, July 2010.

[4] Y. Liu and H. Yu, "Fuzzy control of an underactuated pendulum-driven cart system," International Journal of Advanced Mechatronic Systems, vol. 4, no. 5-6, pp. 260-268, 2012.

[5] A. Knuplez, A. Chowdhury, and R. Svecko, "Modeling and control design for the ball and plate system," in Proceedings of the IEEE International Conference on Industrial Technology (ICIT '03), vol. 2, pp. 1064-1067, Maribor, Slovenia, December 2003.

[6] S. Bi, M. Deng, L. Wang, and Y. Zhao, "Operator-based robust control for MIMO non-linear systems with uncertain hysteresis," International Journal of Advanced Mechatronic Systems, vol. 4, no. 5-6, pp. 212-220, 2012.

[7] M. Deng and N. Bu, "Robust control for nonlinear systems using passivity-based robust right coprime factorization," IEEE Transactions on Automatic Control, vol. 57, no. 10, pp. 25992604, 2012.

[8] M. Deng, S. Wen, and A. Inoue, "Sensorless anti-swing robust nonlinear control for travelling crane system using SVR with generalized Gaussian function and robust right coprime factorization," Transactions of the Society of Instrument and Control Engineers, vol. 47, no. 9, pp. 366-373, 2011.

[9] A. Wang and M. Deng, "Operator-based robust nonlinear tracking control for a human multi-joint arm-like manipulator with unknown time-varying delays," Applied Mathematics \& Information Sciences, vol. 6, no. 3, pp. 459-468, 2012.

[10] S. Wen, M. Deng, S. Bi, and D. Wang, "Operator-based robust nonlinear control and its realization for a multi-tank process by using a distributed control system," Transactions of the Institute of Measurement and Control, vol. 34, no. 7, pp. 891-902, 2011.

[11] M. Jimenez-Lizarraga, M. Basin, and P. Rodriguez-Ramirez, "Robust mini-max regulator for uncertain non-linear polynomial systems," IET Control Theory \& Applications, vol. 6, no. 7, pp. 963-970, 2012.
[12] J. J. Rubio, F. Meléndez, and M. Figueroa, "An observer with controller to detect and reject disturbances," International Journal of Control, vol. 87, no. 3, pp. 524-536, 2014.

[13] J. H. Park and Y. J. Lee, "Robust visual servoing for motion control of the ball on a plate," Mechatronics, vol. 13, no. 7, pp. 723-738, 2003.

[14] D. Casagrande, A. Astolfi, and T. Parisini, "Switching-driving lyapunov function and the stabilization of the ball-and-plate system," IEEE Transactions on Automatic Control, vol. 54, no. 8, pp. 1881-1886, 2009.

[15] S. Awtar, C. Bernard, N. Boklund, A. Master, D. Ueda, and K. Craig, "Mechatronic design of a ball-on-plate balancing system," Mechatronics, vol. 12, no. 2, pp. 217-228, 2002.

[16] X. Fan, N. Zhang, and S. Teng, “Trajectory planning and tracking of ball and plate system using hierarchical fuzzy control scheme," Fuzzy Sets and Systems, vol. 144, no. 2, pp. 297-312, 2004.

[17] M. Zribi, M. Karkoub, and L. Huang, "Modelling and control of two robotic manipulators handling a constrained object," Applied Mathematical Modelling, vol. 24, no. 12, pp. 881-898, 2000.

[18] M. A. Moreno-Armendáriz, E. Rubio, and C. A. Pérez-Olvera, "Design and implementation of a visual fuzzy control in FPGA for the ball and plate system," in Proceedings of the International Conference on Reconfigurable Computing and FPGAs (ReConFig '10), pp. 85-90, Quintana Roo, Mexico, December 2010.

[19] R. M. Murray, Z. Li, and S. S. Sastry, A Mathematical Introduction to Robotic Manipulation, CRC Press, Boca Raton, Fla, USA, 1994.

[20] H. K. Khalil, Nonlinear Systems, Prentice Hall, New York, NY, USA, 3rd edition, 2001.

[21] R. Kelly, "Global positioning of robot manipulators via PD control plus a class of nonlinear integral actions," IEEE Transactions on Automatic Control, vol. 43, no. 7, pp. 934-938, 1998.

[22] J. H. Pérez-Cruz, I. Chairez, J. J. Rubio, and J. Pacheco, "Identification and control of a class of nonlinear systems with nonsymmetric deadzone using recurrent neural networks," IET Control Theory \& Applications, vol. 8, no. 3, pp. 183-192, 2014.

[23] J. J. Rubio, Z. Zamudio, J. Pacheco, and D. Mújica Vargas, "Proportional derivative control with inverse dead-zone for pendulum systems," Mathematical Problems in Engineering, vol. 2013, Article ID 173051, 9 pages, 2013.

[24] J. J. Rubio, "Modified optimal control with a backpropagation network for robotic arms," IET Control Theory \& Applications, vol. 6, no. 14, pp. 2216-2225, 2012.

[25] J. J. Rubio, M. Figueroa, J. H. Pérez Cruz, and J. Rumbo, “Control to stabilize and mitigate disturbances in a rotary inverted pendulum," Mexican Journal of Physical E, vol. 58, no. 2, pp. 107$112,2012$.

[26] C. Torres, J. J. Rubio, C. Aguilar-Ibáñez, and J. H. Pérez-Cruz, "Stable optimal control applied to a cylindrical robotic arm," Neural Computing and Applications, vol. 24, no. 3-4, pp. 937944, 2014.

[27] Terasic Corporation, "1.3 Mega Pixel Digital Camera”.

[28] Pololu, "12 V brushed DC motor with a 50:1 metal gearbox and an integrated quadrature encoder".

[29] Terasic Corporation, "De2 user manual".

[30] A. Buchachia, "Dynamic clustering," Evolving Systems, vol. 3, no. 3, pp. 133-134, 2012.

[31] E. Lughofer, "Single-pass active learning with conflict and ignorance," Evolving Systems, vol. 3, no. 4, pp. 251-271, 2012. 
[32] L. Maciel, A. Lemos, F. Gomide, and R. Ballini, "Evolving fuzzy systems for pricing fixed income options," Evolving Systems, vol. 3, no. 1, pp. 5-18, 2012.

[33] M. Pratama, S. G. Anavatti, P. P. Angelov, and E. Lughofer, "PANFIS: a novel incremental learning machine," IEEE Transactions on Neural Networks and Learning Systems, vol. 25, no. 1, pp. 55-68, 2014. 


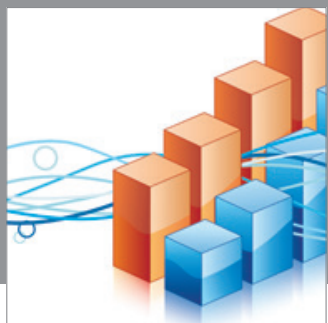

Advances in

Operations Research

mansans

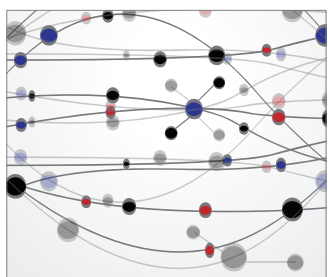

The Scientific World Journal
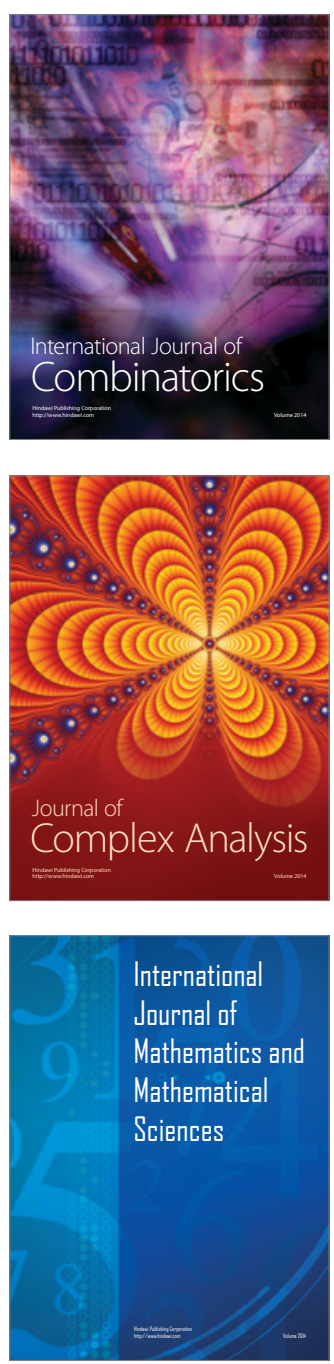
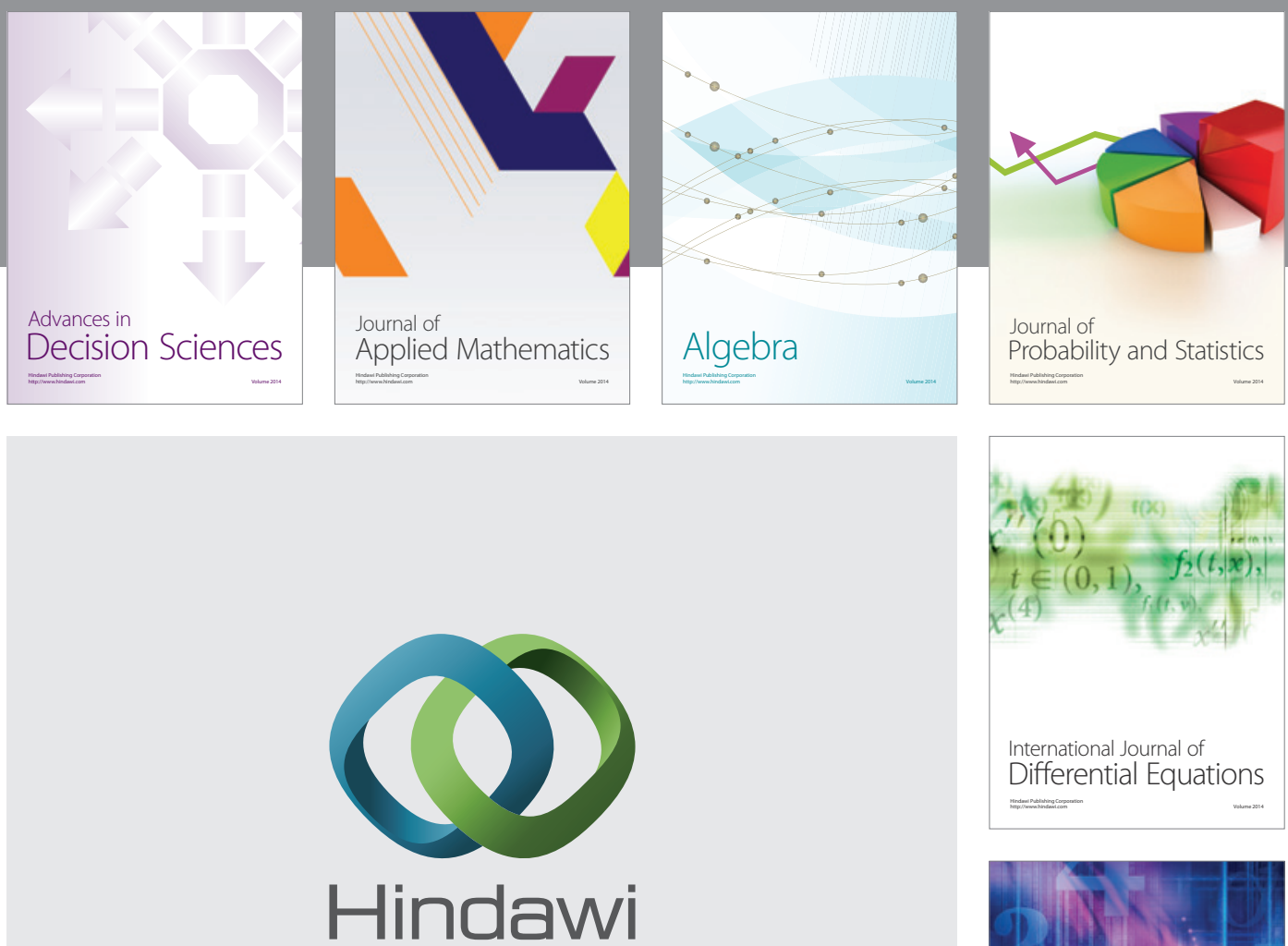

Submit your manuscripts at http://www.hindawi.com
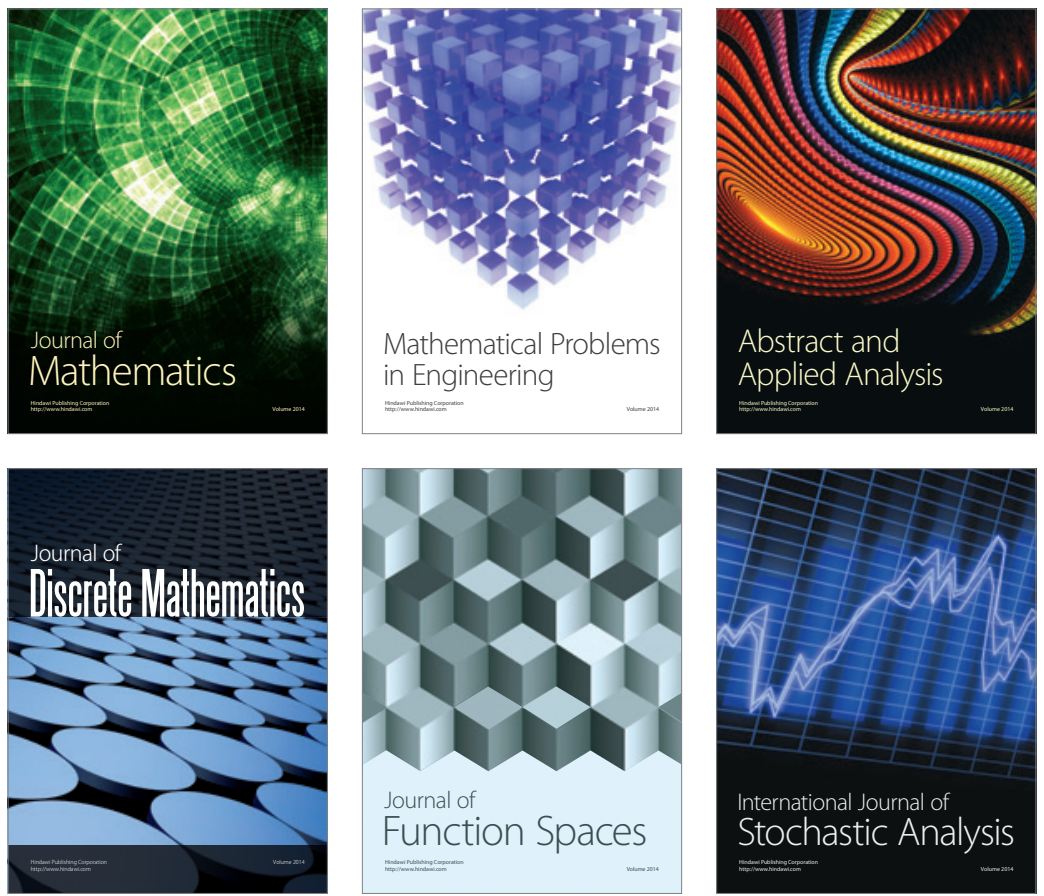

Journal of

Function Spaces

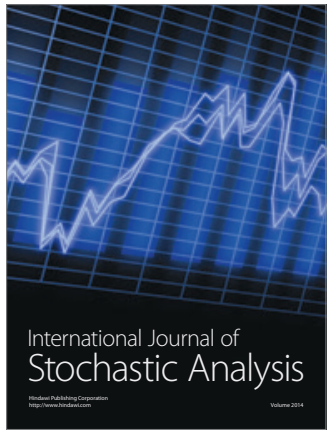

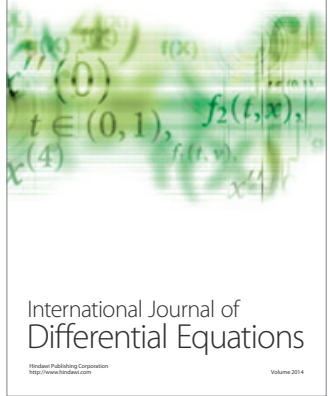
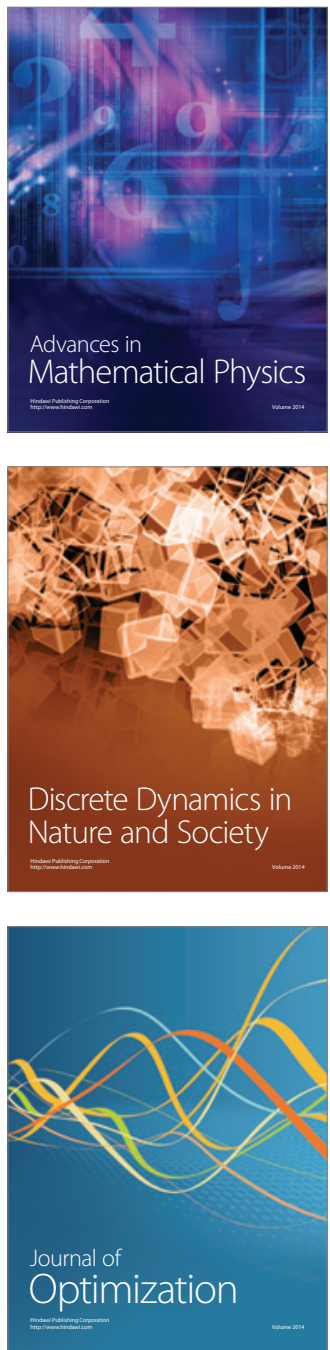